\title{
Conhecimento de cirurgiões-dentistas em relação ao atendimento de diabéticos
}

\section{Dental surgeons knowledge regarding diabetic care}

\author{
1 Rafaella Bastos Leite rrafaella_bastos@hotmail.com \\ 1 Andreza Cristina de Lima Targino Marssoni \\ 1 Danielle do Nascimento Barbosa \\ 2 Kamila Duarte de Sousa \\ 3 Renata de Souza Coelho Soares \\ 3 Raquel Cristina Barboza Gomes
}

\author{
Docente do curso de odontologia pela Universidade Estadual da Paraíba-UEPB. \\ Graduanda em Odontologia pela Universidade Estadual da Paraíba-UEPB. \\ 3 Docente da disciplina de Periodontia pela Universidade Estadual da Paraíba-UEPB.
}

\section{Resumo}

O diabetes mellitus constitui um importante problema de saúde pública, envolvendo altos custos destinados ao tratamento dos pacientes. Esta pesquisa verificou o conhecimento dos cirurgiões-dentistas que trabalham nas Unidades Básicas de Saúde (UBS) e na Equipe de Saúde Bucal (ESB) modalidade I, do município de Campina Grande sobre Pacientes com Diabetes Mellitus (DM), por meio de questionário, próprio e sem critério de exclusão. Foi feita uma entrevista com os cirurgiões-dentistas do município em questão para analisar os seus conhecimentos sobre o tema abordado. Participaram da pesquisa 24 cirurgiões-dentistas que responderam ao questionário o qual abordava 10 questões referentes ao $\mathrm{DM}$, suas características e a sua relação com o atendimento odontológico. Em relação às manifestações orais do paciente portador de diabetes, a doença periodontal foi apontada por $91,6 \%$. Quanto ao atendimento do paciente diabético, $87,5 \%$ afirmaram que o melhor horário para ser atendido seria o turno da manhã, pois eles devem se alimentar normalmente antes da consulta. Em casos de consultas longas, apenas $37,5 \%$ indicaram que deve ser realizado intervalos para que os pacientes possam se alimentar. Os dados obtidos na pesquisa ressaltam que o conhecimento dos cirurgiões-dentistas das UBS do município de Campina Grande é satisfatório, sendo compatível com a necessidade de cuidados para atendimento do paciente diabético na Atenção Primária à Saúde.

\section{Palavras-chave:}

Diabetes Mellitus. Manifestações bucais. Odontologia.

\begin{abstract}
Diabetes mellitus is a major public health problem, involving high costs for the treatment of patients. This study aimed to determine the knowledge of dentists working in primary health care, in the city of Campina Grande, regarding patients with Diabetes Mellitus (DM). Through self-administred questionnaire, without exclusion criteria, an interview was made with the city dentists to analyze their knowledge about the issue adressed. The subjects were 24 professionals, that were able to correctly identify the signs and symptoms of DM and the normal level of glucose in the bloodstream. In relation to the oral manifestations of the patient with diabetes, periodontal disease was reported by $91,6 \%$. As for the diabetic patient care, $87,5 \%$ said that the best time to see diabetic patientes is during the morning and that they should eat normally before the consultation. In cases of long office visit, only $37,5 \%$ indicated that interruptions should be performed so that patients could eat. The data obtained in the study emphasize that the knowledge of dentists from UBS in the city of Campina Grande is satisfactory, such knowledge being compatible with the need for special care for diabetic patients in primary health care.
\end{abstract}

\section{Keywords:}

Diabetes Mellitus. Oral Manifestations. Dentistry.

\section{Como você deve citar?}

LEITE, Rafaella Bastos et al. Conhecimento de cirurgiões-dentistas em relação ao atendimento de diabéticos. Cadernos UniFOA, Volta Redonda, n. 41, p. 119-125, dezembro. 2019. 


\section{INTRODUÇÃO}

É possível conceituar paciente especial como todo indivíduo que possui alteração física, orgânica, intelectual, social ou emocional, podendo ser aguda ou crônica, simples ou complexa, que necessita de educação especial e instruções suplementares, temporária ou definitivamente (PORTER et al., 2014). Dentre as mais variadas necessidades especiais, dar-se-á foco àquelas pertinentes aos pacientes diabéticos.

O Diabetes Mellitus (DM) é um grupo de doenças metabólicas caracterizadas por hiperglicemia e associadas a complicações, disfunções e insuficiência de vários órgãos, especialmente olhos, rins, nervos, cérebro, coração e vasos sanguíneos. Pode resultar de defeitos de secreção e/ou ação da insulina envolvendo processos patogênicos específicos, por exemplo, destruição das células beta do pâncreas (produtoras de insulina), resistência à ação da insulina, distúrbios da secreção da insulina, entre outros (DIRETRIZES DA SOCIEDADE BRASILEIRA DE DIABETES, 2014).

Quanto à classificação, essa patologia é dividida em tipo 1, tipo 2, Diabetes Gestacional e outros tipos específicos. No Diabetes tipo 1, ocorre a destruição das células beta, usualmente levando à deficiência completa de insulina. Já no Diabetes tipo 2, ocorrem graus variados de diminuição de secreção e resistência à insulina, sendo esse tipo o mais frequente (SAMPAIO et al., 2015).

Os sintomas clássicos de DM são: poliúria, polidipsia, polifagia e perda involuntária de peso. Outros sintomas que levantam a suspeita clínica são: fadiga, fraqueza, letargia, prurido cutâneo e vulvar, balanopostite e infecções de repetição. Algumas vezes, o diagnóstico é feito a partir de complicações crônicas, como neuropatia, retinopatia ou doença cardiovascular aterosclerótica. Entretanto o diabetes é assintomático e quando diante da suspeita clínica e identificação dos fatores de risco, deve-se procurar atendimento médico (Ministério da Saúde, 2006). O Cirurgião Dentista (CD) deve estar atento para identificar os sinais e sintomas da DM em pacientes desinformados e não diagnosticados, e preparado para $\mathrm{o}$ atendê-los adequadamente (CANEPPELE et al., 2011).

As manifestações bucais observadas nesses pacientes, embora não específicas dessa doença, têm sua incidência ou progressão favorecida pelo descontrole glicêmico. Os distúrbios da cavidade bucal mais frequentes nos diabéticos são: xerostomia (sensação subjetiva de boca seca que, geralmente, mas não necessariamente, está associada com a diminuição da quantidade de saliva), hiposalivação, síndrome de ardência bucal, glossodinia, distúrbios da gustação, infecções, ulcerações na mucosa bucal, hipocalcificação do esmalte, perda precoce de dentes, dificuldade de cicatrização, doença periodontal, hálito cetônico e líquen plano (FARIA et al., 2015).

O cirurgião dentista tem um papel fundamental na identificação dos sinais e sintomas da DM, possibilitando, assim, o seu correto diagnóstico. É necessário que esses profissionais tenham conhecimento, para que haja um correto planejamento e, consequentemente, um tratamento odontológico mais adequado, ajudando, desse modo, na manutenção da saúde desses pacientes (FARIA et al., 2015).

Portanto, este trabalho tem como objetivo avaliar o conhecimento dos cirurgiões-dentistas no atendimento de pacientes diabéticos, assim como instruir teoricamente os profissionais da rede pública de Campina Grande, favorecendo uma melhor prestação de serviços à comunidade frente ao diagnóstico e tratamento dos pacientes diabéticos. 
Rafaella Bastos Leite / Andreza Cristina de Lima Targino Marssoni | Danielle do Nascimento Barbosa Kamila Duarte de Sousa / Renata de Souza Coelho Soares / Raquel Cristina Barboza Gomes

\section{METODOLOGIA}

O presente trabalho consistiu em uma pesquisa de caráter descritivo, de corte transversal, com aplicação de questionário, caracterizada pela observação e análise do conhecimento dos cirurgiões-dentistas, em atividade na atenção básica do município de Campina Grande, acerca de pacientes diabéticos.

0 universo da amostra foi de $36 \mathrm{CDs}$. A amostra do tipo não probabilística foi obtida por conveniência, ou seja, foi integrada à pesquisa todos os CDs que trabalham em Unidades Básicas de Saúde (UBS) que tenham Equipe de Saúde da família (ESF) modalidade I, que, por livre e espontânea vontade, aceitaram participar desta pesquisa e assinarem um Termo de Consentimento Livre e Esclarecido (TCLE), tendo a amostra final 24 CDs.

O instrumento de coleta de dados foi um questionário composto por 10 (dez) questões, todas objetivas, no qual os CDs poderiam assinalar mais de uma alternativa. Os dentistas analisados responderiam apenas após terem sido explicados sobre a finalidade da pesquisa e, caso aceitassem, terem assinado o TCLE.

A coleta fora realizada nas próprias Unidades Básicas de Saúde, entre o período de outubro e novembro de 2014. A escolha do local foi feita com a finalidade de dinamizar o preenchimento do questionário, assim como impedir a consulta de quaisquer materiais, buscando a maior fidedignidade da pesquisa.

O presente estudo recebeu a aprovação do Comitê de Ética em Pesquisa com Seres Humanos da Universidade Estadual da Paraíba (Brasil), sob processo número 35555314.1.0000.5187, em complacência com a Resolução 466/12 do Conselho Nacional de Saúde/Ministério da Saúde e RESOLUÇÃO/UEPB/ CONSEPE/10/2001. Os direitos de todos os participantes foram protegidos.

\section{RESULTADOS}

Do total de 36 Cirurgiões Dentistas que atuam na atenção básica do Sistema Único de Saúde (SUS) do município de Campina Grande - Paraíba, 24 profissionais participaram desta pesquisa, o que corresponde a uma taxa de resposta de $66,6 \%$.

Dos 24 cirurgiões-dentistas entrevistados, apenas 23 responderam à questão relativa ao tempo de formação. Destes, 19 (79,1\%) já haviam se formado há mais de 10 anos, 03 (12,5\%) eram formados de 07 a 10 anos e apenas 01 dentista (4,1\%) se formara entre 04 e 07 anos.

Os resultados mostram que a maioria dos CDs (18 - 75\%) acertaram o índice normal de glicose na corrente sanguínea em jejum; $22(91,6 \%)$ assinalaram corretamente quais os principais sinais e sintomas que levam à suspeita de DM, como pode ser observado na tabela 1.

Tabela 1 - Avaliação das respostas dos dentistas da Estratégia da Saúde da Família relacionadas ao nível de glicose normal e sinais e sintomas de DM

\begin{tabular}{llll}
\hline \multicolumn{1}{c}{ Questão } & $n *$ & Correto & Incorreto \\
\hline $\begin{array}{l}\text { Qual o índice normal de glicose na corrente sanguínea em } \\
\text { jejum }\end{array}$ & 24 & $18(75 \%)$ & $06(25 \%)$ \\
São considerados os principais sinais e sintomas de DM & 24 & $22(91,6 \%)$ & $2(8,3 \%)$ \\
\hline *número
\end{tabular}

*número de profissionais que responderam a estas questões. 
Em relação às manifestações orais do paciente portador de DM, a doença periodontal foi apontada por $22(91,6 \%)$ dos entrevistados.

Na questão sobre o horário mais recomendado para o atendimento dos pacientes diabéticos, 21 $(87,5 \%)$ profissionais responderam que seria no período da manhã e $3(12,5 \%)$ responderam que seria no período da tarde.

Sobre a conduta que deve ser adotada pelo paciente diabético, 21 (87,5\%) dos CDs responderam que eles devem se alimentar normalmente e $3(12,5 \%)$ responderam que devem se alimentar pouco.

No caso de consultas longas, 11 profissionais $(45,8 \%)$ responderam que deveriam realizar mensurações frequentes de glicemia. Apenas $9(37,5 \%)$ dos entrevistados recomendaram a realização de intervalos para que os pacientes possam se alimentar, 3 (12,5\%) CDs não acham necessário realizar interrupções e $2(8,3 \%)$ participantes não responderam a essa questão.

A tabela 2 demonstra a distribuição relativa das respostas dos cirurgiões-dentistas para as questões referentes ao melhor horário de atendimento, conduta que deve ser adotada pelo paciente diabético antes das consultas e conduta do CD frente a atendimentos longos.

Tabela 2 - Avaliação das respostas dos CDs referente ao melhor horário de atendimento, conduta que deve ser adotada pelo paciente diabético antes das consultas e conduta do $C D$ frente a atendimentos longos.

\begin{tabular}{llll}
\hline \multicolumn{1}{c}{ Questão } & $\mathrm{n}^{*}$ & Correto & Incorreto \\
\hline $\begin{array}{l}\text { Qual o horário mais recomendado para a consulta } \\
\text { odontológica do paciente diabético? }\end{array}$ & 24 & $21(87,5 \%)$ & $03(12,5 \%)$
\end{tabular}

\begin{tabular}{|c|c|c|c|}
\hline $\begin{array}{l}\text { Antes do atendimento, no qual será feito o uso de } \\
\text { anestésico local, qual procedimento adotado para o } \\
\text { paciente diabético? }\end{array}$ & 24 & $21(87,5 \%)$ & $03(12,5 \%)$ \\
\hline Qual a melhor conduta profissional frente a consultas de & 22 & $09(37,5 \%)$ & $18(66,6 \%)$ \\
\hline
\end{tabular}

*número de profissionais que responderam a estas questões.

Em relação ao anestésico de escolha para uso em pacientes diabéticos, 11 (45,8\%) dos entrevistados escolheram a opção correta. 0 anestésico mais utilizado foi o cloridrato de prilocaína com felipressina. Em seguida, as opções mais escolhidas pelos CDs foram, respectivamente, mepvacaina $(29,1)$, lidocaína + epinefrina $(16,6 \%)$ e lidocaína $(16,6 \%)$.

No questionamento sobre situações que necessitam de profilaxia antibiótica para o paciente diabético, $17(70,8 \%)$ responderam que é indicado, quando esses pacientes apresentam infecções frequentes, 20 (83,3\%) responderam que é indicado em casos de DM descompensado, 2 (8,3\%) responderam que é indicado frente a diabetes compensada e $8,3 \%$ responderam em caso de hipoglicemia.

Quanto aos sinais e sintomas de hipoglicemia, as principais respostas citadas pelos CDs foram suor frio $95,8 \%$, fraqueza $91,6 \%$ e palidez $87,5 \%$. 
Rafaella Bastos Leite / Andreza Cristina de Lima Targino Marssoni / Danielle do Nascimento Barbosa Kamila Duarte de Sousa / Renata de Souza Coelho Soares / Raquel Cristina Barboza Gomes

\section{DISCUSSÃo}

O DM é considerado um grave problema de saúde pública devido ao aumento de sua incidência e por ser uma doença sistêmica, tendo influência em todo o organismo, inclusive na cavidade oral, portanto, é importante que o $\mathrm{CD}$ faça parte da equipe multiprofissional que atende a esses pacientes (FARIA et al., 2015).

Os critérios diagnósticos para DM adotado pela American Diabetes Association (ADA), posteriormente aceito pela Organização Mundial da Saúde (OMS) e pela Sociedade Brasileira de Diabetes (SBD) consistem em sintomas de poliúria, polidipsia e perda ponderal acrescidos de glicemia casual acima de $200 \mathrm{mg} / \mathrm{dl}$; glicemia de jejum igual ou maior $126 \mathrm{mg} / \mathrm{dl}$ ( 7 milimols) e glicemia de 2 horas pós-sobrecarga de $75 \mathrm{~g}$ de glicose acima de $200 \mathrm{mg} / \mathrm{dl}$. O valor de corte inferior a $100 \mathrm{mg} / \mathrm{dL}$ para glicemia de jejum normal é adotado pela ADA e pela SBD e recomendada pela Federação Internacional de Diabetes (IDF) e níveis de glicose acima desse valor são consideradas como categorias de glicemia de jejum alterada e tolerância à glicose diminuída (MATSUMOTO et al., 2012). No nosso estudo, a maioria dos participantes (75\%) acertaram a questão referente ao nível normal de glicose em jejum, escolhendo a opção que continha valor abaixo de $100 \mathrm{mg} / \mathrm{dl}$. Já $25 \%$ consideraram como nível normal de glicose em jejum o valor até $110 \mathrm{mg} / \mathrm{dl}$. Em relação aos sinais e sintomas que levam à suspeita de DM, $91,6 \%$ dos entrevistados identificaram corretamente.

A doença periodontal é a complicação oral mais frequente, sendo considerada pela OMS a sexta complicação clássica do diabetes (FARIA et al., 2015). Vários mecanismos estão envolvidos na fisiopatogenia da doença periodontal associada à DM (FARIA et al., 2015). A susceptibilidade e progressão da infecção dos tecidos de proteção e sustentação dos dentes estão relacionadas com o descontrole metabólico, produtos de glicosilação avançados formados a partir da ligação da glicose a proteínas estruturais, deficiente resposta imune, redução da quimiotaxia de neutrófilos, função reduzida dos fibroblastos, alterações dos vasos sanguíneos, tecido conjuntivo, composição salivar e genética, como herança de determinados antígenos de histocompatibilidade (PORTER et al., 2014).

Outros distúrbios da cavidade bucal frequentes nos diabéticos são: xerostomia, hiposalivação, síndrome de ardência bucal, glossodinia, distúrbios da gustação, infecções, ulcerações na mucosa bucal, hipocalcificação do esmalte, perda precoce de dentes, dificuldade de cicatrização, doença periodontal, hálito cetônico e líquen plano (FARIA et al., 2015).

As manifestações bucais apontadas pelos entrevistados no presente estudo foram de doença periodontal (91,6\%), xerostomia (54\%), candidíase (37,5\%) síndrome de ardência bucal (25\%) e hiperplasia fibrosa inflamatória (20,8\%). Em relação à hiperplasia fibrosa inflamatória, não foram encontrados estudos que relacionam esse tipo de lesão ao DM.

No que se refere ao melhor horário de atendimento para esses pacientes, deve-se escolher o horário no período da manhã, uma vez que coincide com o café da manhã e aplicação de insulina (MILTON et al., 2012; TSCHIEDEL et al., 2008; TORRES et al., 2013). Antes do atendimento, o paciente deve se alimentar normalmente para que não ocorra hipoglicemia. Caso se manifestem sintomas de hipoglicemia, sem perda de consciência durante o tratamento, o cirurgião-dentista deve oferecer sucos ou doces para que o nível de glicose se eleve (TORRES et al., 2013). No atendimento de pacientes diabéticos, procura-se sempre realizar procedimentos curtos ou com intervalos, para que o paciente possa se alimentar, evitando a hipoglicemia (TSCHIEDEL et al., 2008). Na avaliação dos questionários, foi verificado que 21 dos entrevistados (87,5\%) acertaram o melhor horário de atendimento, 21 profissionais (87,5\%) sabem que os pacientes devem se alimentar normalmente para o atendimento. Já relacionado às consultas longas para paciente diabéticos, apenas 9 dos CDs (37,5\%) recomendam intervalos para alimentação. Resultados semelhantes foram encontrados em outro estudo semelhante (CANEPPELE et al., 2011). 
Quanto aos sinais e sintomas de hipoglicemia, os CDs citaram suor frio (95,8\%), fraqueza (91,6\%), palidez $(87,5 \%)$, confusão mental $(66,6 \%)$, fome $(45,8 \%)$, hálito cetônico $(41,6 \%)$, sonolência $(37,5 \%)$, vômito e náuseas $(4,1 \%)$. Segundo a literatura, os sinais são adrenérgicos (tremor, sudorese, palidez, taquicardia, palpitação, fome) e neuroglicopênicos (cefaleia, tontura, sonolência, irritabilidade, fraqueza, confusão mental, visão turva, incoordenação motora, desmaio, convulsão e coma) (FARIA et al., 2015; MELGAÇO et al., 2002). Parte dos entrevistados assinalaram sinais e sintomas de hiperglicemia.

Em relação à profilaxia antibiótica, existindo processo infeccioso com controle metabólico pobre e risco de infecção secundária, deverá ser feita a antibioticoterapia (MELGAÇO et al., 2002). No questionamento sobre situações que necessitam de profilaxia antibiótica para o paciente diabético, $70,8 \%$ dos entrevistados responderam que deve-se prescrever, em casos onde o paciente diabético apresenta o quadro de infecções frequente, $83,3 \%$ dos CDs responderam que deve-se prescrever em caso de DM descompensado. Já $8,3 \%$ dos profissionais responderam que seria indicada profilaxia antibiótica frente a pacientes com diabetes compensada e 8,3\% responderam em caso de hipoglicemia, corroborando com outro estudo realizado (MELGAÇO et al., 2002), sugerindo falta de leitura e estudo sobre o tema por parte dos CDs.

Quanto ao anestésico de escolha, obtivemos resultado semelhante a outra pesquisa já realizada, na qual grande parte dos entrevistados $(45,8 \%)$ escolheram o cloridrato de prilocaína com felipressina como anestésico de escolha para pacientes diabéticos (CANEPPELE et al., 2011). Em pacientes compensados, os anestésicos locais com adrenalina ou noradrenalina podem ser usados sem problemas. Por outro lado, em pacientes com descontrole metabólico, a indicação de anestésico com adrenalina é controversa (TÓFOLI et al., 2005). Alguns autores recomendam evitar uso de soluções com vasoconstrictores à base de adrenalina e noradrenalina, pois elas promovem a quebra de glicogênio em glicose, aumentando ainda mais os níveis de glicose circulante (MELGAÇO et al., 2002; TÓFOLI et al., 2005). Nesses casos, os autores recomendam usar preparados sintéticos (felipressina) ou usar anestésicos sem vasoconstrictores (TÓFOLI et al., 2005).

Embora se tenha obtido respostas satisfatórias por parte dos entrevistados, observa-se a necessidade de um melhor aprimoramento dos profissionais, a fim de que a população possa se encontrar mais bem assistida, já que, ao cuidar da saúde bucal dos pacientes portadores dessa doença, o CD contribui na manutenção da saúde geral desses pacientes.

\section{CONCLUSÃO}

Os dados obtidos na pesquisa ressaltam que o conhecimento dos cirurgiões-dentistas das Unidades Básicas de Saúde do município de Campina Grande é satisfatório, compatível com a necessidade de cuidados para atendimento do paciente diabético na Atenção Primária à Saúde. Entretanto, como o conhecimento é muito dinâmico, enfatiza-se que os profissionais precisam ser estimulados a um constante aprimoramento, para realizar atendimentos com mais qualidade. 
Rafaella Bastos Leite / Andreza Cristina de Lima Targino Marssoni / Danielle do Nascimento Barbosa Kamila Duarte de Sousa / Renata de Souza Coelho Soares / Raquel Cristina Barboza Gomes

\section{REFERÊNCIAS}

CANEPPELE, T.M.F. et al. Conhecimento do cirurgião-dentista sobre o atendimento a pacientes especiais: hipertensos diabéticos e gestantes. Rev. Odontologia, v. 1, p.31-41, 2011.

DIRETRIZES DA SOCIEDADE BRASILEIRA DE DIABETES: 2013-2014/Sociedade Brasileira de Diabete. São Paulo: AC Farmacêutica; 2014.

FARIA, H.T.G.; VERAS, V.S.; XAVIER, A.T.F.; TEIXEIRA, C.R.S.; ZANETTI, M.L.; SANTOS, M.A. Qualidade de vida de pacientes com diabetes mellitus antes e após participação em programa educativo. Rev Esc Enferm USP, v.47, p.348-54, 2015.

MATSUMOTO, P.M.; BARRETO, A.R.B.; SAKATA, K.N.; SIQUEIRA, Y.M.C.; ZOBOLI EL, C.P.; FRACOLLI, L.A. A educação em saúde no cuidado de usuários do Programa Automonitoramento Glicêmico. Rev Esc Enferm USP, v.46:761-5, 2012.

MELGAÇO, C.A. Diabetes melito e a doença periodontal: revisão de literatura. J Bras Endo/Perio, v. 3, p. 100-4, 2002.

MILTON, K.P.C.T. Critical Appraisal Skills Programme. Oxford: CASP UK, 2012.

PORTER, R.S.; KALAN JL. Manual Merck Diagnóstico e Tratamento. 19. ed. São Paulo: Roca; 2014.

SAMPAIO, H.A.C.; CARIOCA, A.A.F.; SABRY, M.O.D.; SANTOS, P.M.; COELHO, M.A.M.; PASSAMAI, M.P.B. Letramento em saúde de diabéticos tipo 2: fatores associados e controle glicêmico. Ciênc Saúde Colet, v.20, p.865-74, 2015.

TÓFOLI, G.R. et al. Tratamento odontológico em pacientes com diabetes mellitus. R. Assoc. Paul. Cir. Dent., São Paulo, v.59, p.306-310, 2005.

TORRES, H.C.; REIS, I.A.; FARIA, P.; ROQUE, C. Telephone-based monitoring: an educational strategy for diabetes self-care at the primary health care level. Cienc Enferm v.19, p.95-105, 2013.

TSCHIEDEL B et al. Organização de um serviço de assistência ao paciente com diabetes melito tipo 1. Arq Bras Endocrinol Metab, v. 52, p. 34-39, 2008. 